\title{
DIAGNOSTIC AGREEMENT BETWEEN PANORAMIC RADIOGRAPHS AND COLOR DOPPLER IMAGES OF CAROTID ATHEROMA
}

\author{
Claudia Maria ROMANO-SOUSA ${ }^{1}$, Laís KREJCI ${ }^{2}$, Flavilene Marchioro Martins MEDEIROS ${ }^{2}$, Ricardo Gomes GRACIOSA- \\ FILHO $^{3}$, Maria Fernanda Fonseca MARTINS ${ }^{2}$, Vanessa Novaes GUEDES ${ }^{2}$, Marlene FENYO-PEREIRA ${ }^{4}$
}

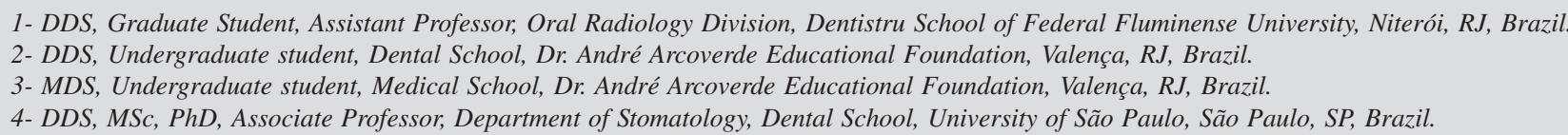

Corresponding address: Marlene Fenyo-Pereira - Faculdade de Odontologia da Universidade de São Paulo Departamento de Estomatologia - Disciplina de Radiologia - Av. Prof. Lineu Prestes, 2227 - 05508-900 - Cidade Universitária - São Paulo,SP - Brasil. Phone: +55-11-30917831- Fax: +55-1130917899 - e-mail:mfenyo@usp.br

Received: September 26, 2007 - Modification: March 13, 2008 - Accepted: August 13, 2008

\begin{abstract}
$T_{\text {he }}$

he aim of this study was to investigate the agreement between diagnoses of calcified atheroma seen on panoramic radiographs and color Doppler images. Our interest stems from the fact that panoramic images can show the presence of atheroma regardless of the level of obstruction detected by color Doppler images. Panoramic and color Doppler images of 16 patients obtained from the archives of the Health Department of the city of Valença, RJ, Brazil, were analyzed in this study. Both sides of each patient were observed on the images, with a total of 32 analyzed cervical regions. The level of agreement between diagnoses was analyzed using the Kappa statistics. There was a high level of agreement, with a Kappa value of 0.78. In conclusion, panoramic radiographs can help detecting calcifications in the cervical region of patients susceptible to vascular diseases predisposing to myocardial infarction and cerebrovascular accidents. If properly trained and informed, dentists can refer their patients to a physician for a cardiovascular evaluation in order to receive proper and timely medical treatment.
\end{abstract}

Key words: Radiography, panoramic. Carotid artery, external. Carotid artery diseases. Calcinosis.

\section{INTRODUCTION}

Increased blood pressure, high serum levels of cholesterol or carbohydrates, and other factors may lead to endothelial damage and consequent atherogenesis ${ }^{6}$. The presence of carotid atheroma is associated with the severity of coronary artery disease (CAD) and cerebrovascular accident (CVA) or cerebral infarction.

Among the different methods to diagnose atherosclerotic diseases, angiography is considered the gold standard. However, because it is an invasive method, complications may occur ${ }^{5,24,25}$. Therefore, color Doppler imaging, also called laser Doppler fluxometry or duplex scan, has been increasingly used to diagnose atheroma because it is a fast, accurate and painless method of diagnosis. Color Doppler can be considered as a gold standard because the results obtained with this imagining method are similar to those obtained with angiography, with the advantage that color Doppler is a noninvasive method ${ }^{7,15,16,19,23}$.

Panoramic radiographs, used as a complementary examination resource by dentists, can often show the presence of carotid atheroma ${ }^{2,22}$. Most studies found in dental literature report that calcifications between the $2 \mathrm{nd}$, 3rd and 4th vertebrae first seen on panoramic radiographs are further confirmed as atheroma by color Doppler ${ }^{3,8,10,20}$.

Studies have investigated the possibility of identifying carotid artery calcifications on panoramic radiographs, on many occasions especially in asymptomatic cases. Therefore, patients can be referred to a cardiologist or a neurologist for further investigation ${ }^{9,12}$.

The use of panoramic images to assess carotid atheroma is important because they often show the presence of calcifications. Panoramic radiographs are easy to take and have a low cost compared to other imaging methods. In addition, a complete radiographic examination of the both arches can be carried out with only one $\mathrm{x}$-ray exposure and a relatively lower radiation dose $\mathrm{e}^{2,21}$.

Our interest stems from the fact that panoramic radiographs can show the presence of carotid calcification regardless of the degree of obstruction. Therefore, the purpose 
of this study was to investigate the agreement between diagnoses of calcified atheroma seen on panoramic radiographs and color Doppler images.

\section{MATERIAL AND METHODS}

\section{Material}

This research was independently reviewed and approved by the Research Ethics Committee of the Medical School of Valença, Rio de Janeiro, RJ, Brazil.

Color Doppler and panoramic images of 16 patients obtained from the archives of the Health Department of the city of Valença, Brazil, were analyzed in this study. Both sides of each patient were observed on the images, with a total of 32 analyzed cervical regions.

The criteria for selecting the images considered the date of the examinations and were selected radiographic and color Doppler images taken on the same day and interpreted by different radiologists who were responsible for the clinics accredited by the Health Department of the city of Valença, RJ, Brazil.

\section{Methods}

The panoramic radiographs were analyzed by a dental radiologist with 20 years of experience. The radiographic findings of calcifications in the cervical region (Figure 1) were compared to the results of color Doppler ultrasonography (Figure 2). Both sides of each patient were examined.

After analyzing the panoramic images, it was verified whether a positive or negative diagnosis of calcification was made and the results were compared to those obtained from the color Doppler ultrasound images, which were analyzed by the same ultrasound medical specialist.

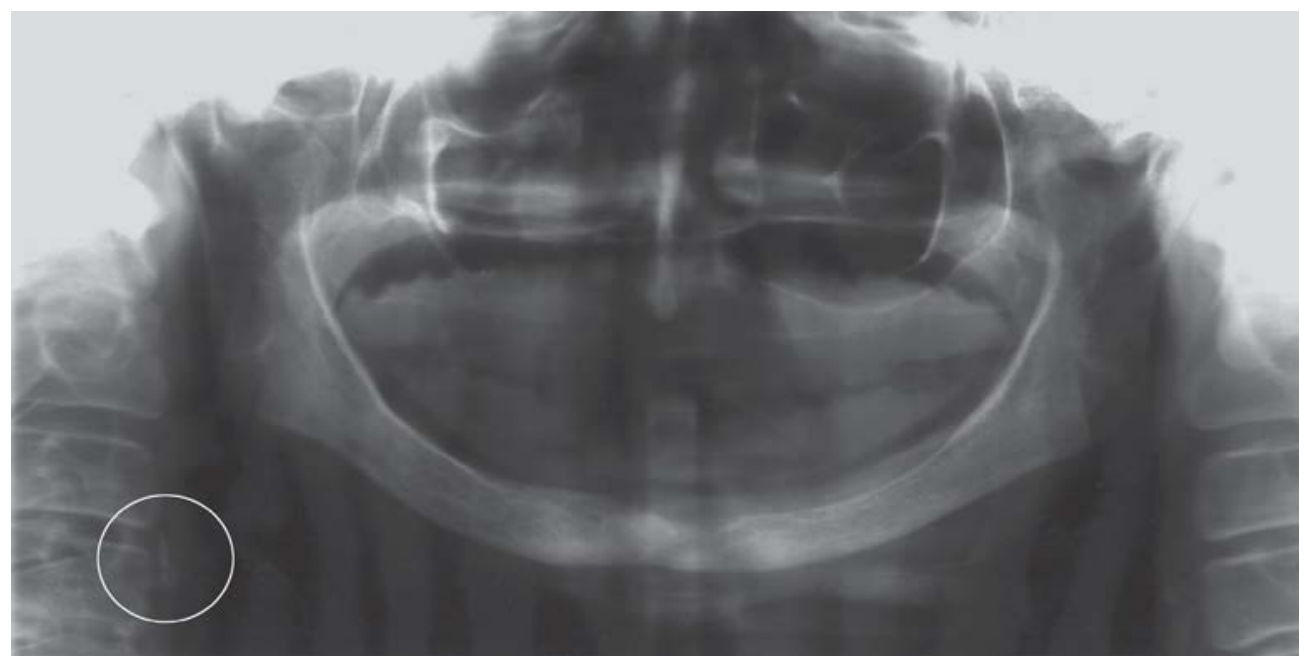

FIGURE 1- Panoramic radiography shows a calcification in the cervical region on the right side

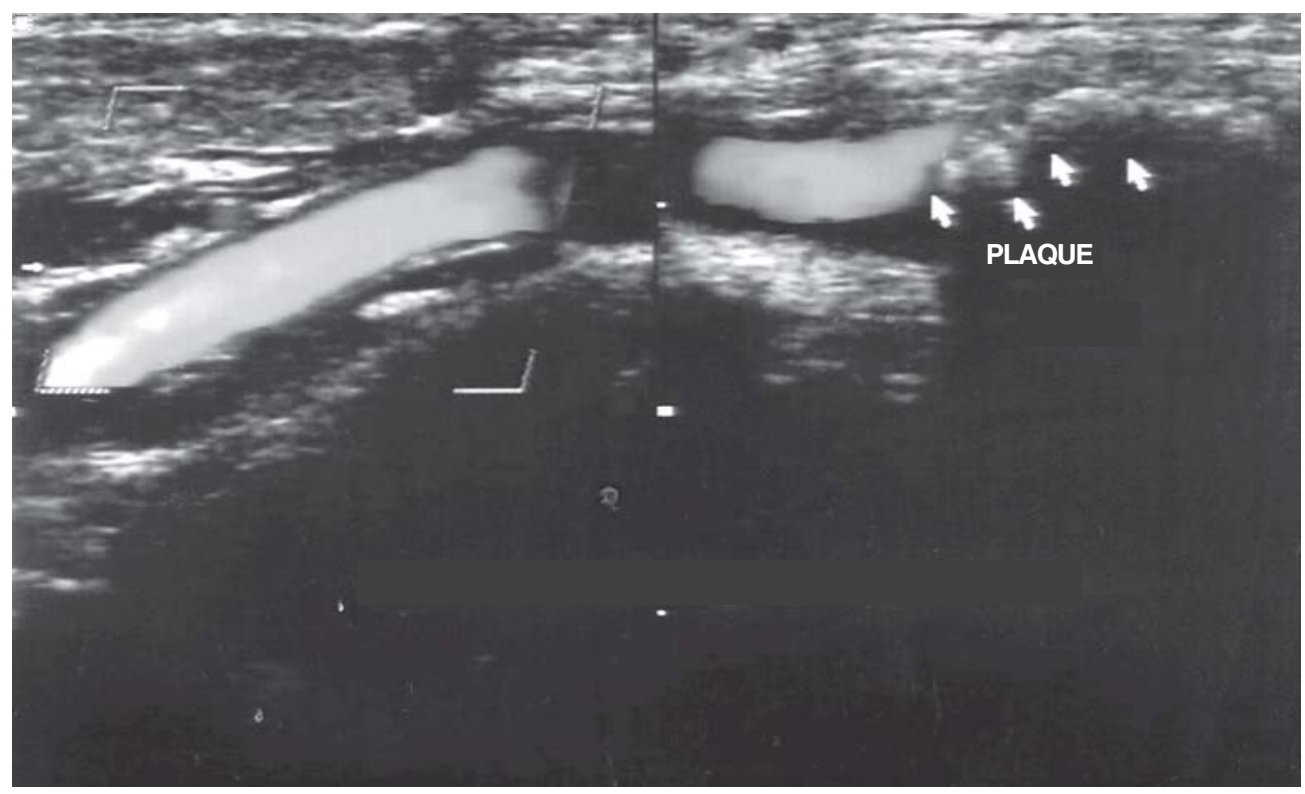

FIGURE 2- Carotid stenosis in the left internal carotid artery by color Doppler images 


\section{RESULTS}

Figure 3 shows the comparison between the diagnoses of unilateral and/or bilateral carotid artery calcifications after analysis of panoramic radiographs and color Doppler images.

The results were distributed into two groups, subdivided into two subgroups: 1. Agreement between diagnoses, which included all cases with similar diagnoses after examination of both panoramic and color Doppler images; 2. Disagreement between diagnoses, which included all cases with different diagnoses after examination of both panoramic and color Doppler images.
Some authors ${ }^{23}$ do not indicate the use of panoramic radiographs to detect carotid artery calcifications arguing that this type of method of diagnosis has low sensitivity (31.1\%). In the present study, it was not our intention to indicate panoramic radiography as the exam of choice for detecting calcification in the carotid artery. Instead, we intended to demonstrate that panoramic radiography is an important adjuvant for enabling patient general evaluation by the dentist, who can adequately refer patients to physicians if a suspicion of atheroma or other pathology arises.

The calcification seen on the panoramic radiographic image has been described as a nodular area of radiopacity. Radiographically, we can observe one or various vertical linear

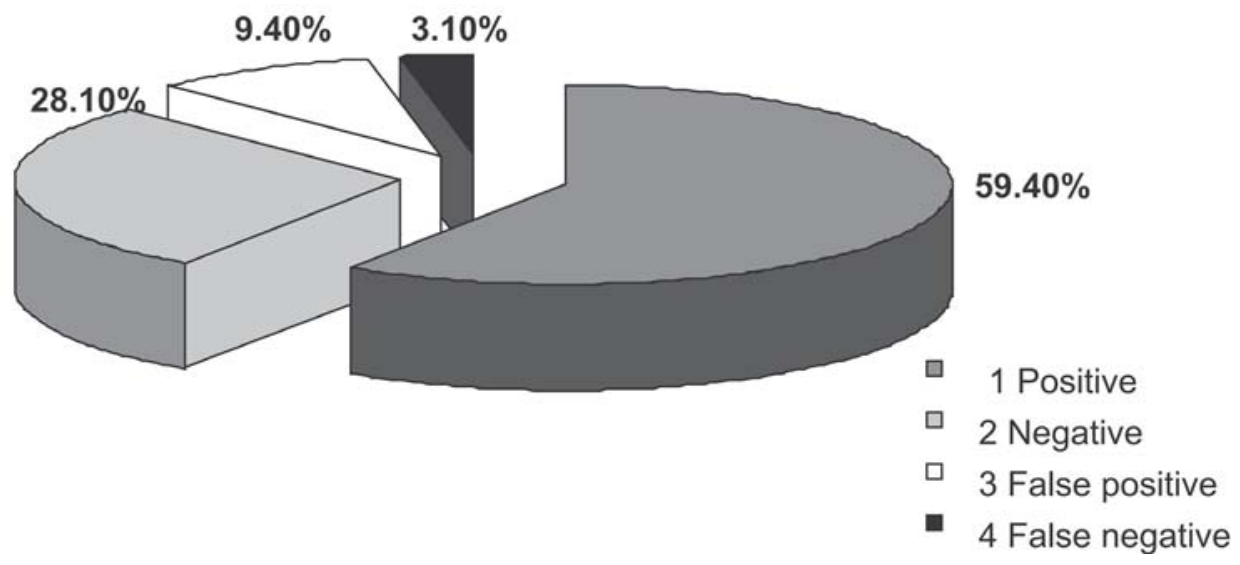

FIGURE 3- Positive, negative, false-positive and false-negative diagnosis

\section{DISCUSSION}

Differential diagnosis of the structures that constitute the carotid region is extremely important. It is necessary to have extensive knowledge of all anatomical structures that may produce radiographic images similar to carotid atheroma, such as the hyoid bone, the epiglottis, calcified stylomandibular and stylohyoid ligaments, and the triticeal cartilage, as well as pathological processes such as sialoliths, phleboliths, and calcified lymph nodes. The radiologists responsible for the analysis of the images must be able to identify anatomical structures and pathological calcifications in soft tissues in the head and neck region, discerning their location ${ }^{1,3,11-13,18}$.

Previous studies have demonstrated that panoramic radiographs of patients undergoing dental treatment can often reveal the presence of carotid atheroma, later confirmed by color Doppler ultrasonography $y^{2,8,12,14}$.

Carotid artery calcifications may lead to cerebrovascular accidents (CVA), the third major cause of death in Australia and the USA ${ }^{1}$. CVA may also affect a person's functional ability, and the physical and psychological rehabilitation is difficult and expensive ${ }^{17}$. It is therefore important that panoramic radiographs are used to help establishing an early detection and prevention cerebrovascular or coronary artery diseases an early diagnosis of carotid artery calcifications. The dentist can help save lives with a radiograph taken for dental care purposes ${ }^{2}$. areas of radiopacity, with a long or triangular shape and various sizes. These areas are observed approximately 1.5 to $4.0 \mathrm{~cm}$ from the mandibular angle and/or posterior to the mandible and the $2 \mathrm{nd}, 3 \mathrm{rd}$, and 4 th vertebrae, adjacent to the intervertebral space s,8,9,10,20. $^{3}$.

The vertebral region is not currently investigated by dentists. However, if dentists were previously trained to identify calcifications in the cervical region on panoramic images and confirm the presence of atheroma by means of other imagining methods, they would help reducing the incidence of cerebrovascular or coronary artery diseases ${ }^{2}$. A study investigating the panoramic images of 1,548 neurologically asymptomatic patients aged 50 or older showed the presence of carotid atheroma in 65 patients, later confirmed by color Doppler ${ }^{14}$. The study showed that early or advanced stages of atherosclerotic disease can be identified through careful analysis of panoramic radiographs by dentists.

There is a report in the literature of an asymptomatic patient whose panoramic radiograph showed calcifications in the carotid region, and color Doppler later showed over $90 \%$ bilateral stenosis in the external carotid artery of the patient $^{4}$.

The purpose of our study was to assess the relationship between calcifications seen on panoramic images of the cervical region and by the analysis of color Doppler images. A total of 32 regions were bilaterally examined in 16 patients who had undergone panoramic x-ray exposure and color 
Doppler ultrasound. Calcifications were seen on both panoramic and color Doppler images of 19 regions (59.4\%), which was considered a positive diagnosis. No atheroma was seen on panoramic or color Doppler images of 9 regions $(28.1 \%)$, which were considered a negative diagnosis. Calcifications were seen on panoramic images but not confirmed by color Doppler in 3 regions $(9.4 \%)$, which was considered a false-positive diagnosis. This might be related to anatomical structures or pathological calcifications in soft tissues in the head and neck region ${ }^{1,3,11,12,13,18}$. In 1 case $(3.1 \%)$ color Doppler showed the presence of a calcification that had not been seen on the panoramic image. This was considered a false-negative diagnosis, and might be related to the degree of calcification. We reviewed the related literature and found no reports on the degree of calcification necessary to produce a radiopaque image specific for atheroma.

\section{CONCLUSION}

It may be concluded that panoramic radiographs may help detecting calcifications in the cervical region of patients susceptible to vascular diseases predisposing to myocardial infarction and cerebrovascular accidents and refer these patients to a neurologist or cardiologist. Since the panoramic radiography is a common, non-expense exam, the present study and similar ones might widen the perspectives to a possible interdisciplinary partnership approach where properly trained and informed dentists can advise their patients as to the necessary steps to be taken in order to receive a correct medical treatment.

\section{REFERENCES}

1- Almog DM, Tsimidis K, Moss ME, Gottilieb RH, Carter LC. Evaluation of a training program for detection of carotid artery calcifications on panoramic radiographs. Oral Surg Oral Med Oral Pathol Oral Radiol Endod. 2000;90(1):111-7.

2-Almog DM. Utility of panoramic radiographs in detecting cervical calcified carotid atheroma. (Letters to the editor). . Oral Surg Oral Med Oral Pathol Oral Radiol Endod. 2007;104(4):451-2.

3- Carter LC. Discrimination between calcified triticeous cartilage and calcified carotid atheroma on panoramic radiography. Oral Surg Oral Med Oral Pathol Oral Radiol Endod. 2000;90(1):108-10.

4- Carter LC, Tsimidis K, Fabiano J. Carotid calcifications on panoramic radiography identify an asymptomatic male patient at risk for stroke. Oral Surg Oral Med Oral Pathol Oral Radiol Endod. 1998;85(1):119-22.

5- Cinat M, Lane CT, Phan H, Lee A, Wilson SE, Gordon I. Helical CT angiography in the preoperative evaluation of carotid artery stenosis. J Vasc Surg. 1998;28(2):290-300.

6- Fabris F, Zanocchi M, Bo M, Fonte G, Poli L, Bergoglio I, et al. Carotid plaque, aging and risk factors. A study of 457 subjects. Stroke. 1994;25(6):1133-40.

7- Frauchiger B, Schimid HD, Roedel C, Moosmann P, Staub D. Comparison of carotid arterial resistive indices with intima-media thickness as sonographic markers of atherosclerosis. Stroke. 2001;32(4):836-41.
8- Freymiller EG, Sung EC, Friedlander AH. Detection of radiation-induced atheromas by panoramic radiography. Oral Oncology. 2000;36(2):175-9.

9- Friedlander AH. Identification of stroke-prone patients by panoramic and cervical spine radiography. Dentomaxillofac Radiol. 1995;24(3):1604

10- Friedlander AH. Panoramic radiography: the differential diagnosis of carotid artery atheromas. Spec Care Dentist. 1995;15(6):223-7.

11- Friedlander AH, Baker D. Panoramic radiography: an aid in detecting patients at risk of cerebrovascular accident. J Am Dent Assoc. 1994;125:1598-603

12- Friedlander AH, Lande A. Panoramic radiographic identification of carotid arterial plaques. Oral Surg Oral Med Oral Pathol. 1981;52(1);10204.

13- Friedlander AH, Friedlander IK, Yueh R, Littner MR. The prevalence of carotid atheromas seen on panoramic radiographs of patients with obstructive sleep apnea and their relation to risk factors for atherosclerosis. J Oral Maxillofac Surg. 1999;57(5):516-21.

14- Friedlander AH, Garrett NR, Chin EE, Baker JD. Ultrasonographic confirmation of carotid artery atheromas diagnosed via panoramic radiography. J Am Dent Assoc. 2005;136(5):635-40.

15- Handa N, Matsumoo M, Hougaku H. Ultrasonic evaluation of early carotid atherosclerosis. Stroke. 1990;21(11):1567-72.

16- Johnson M, Wilkinson I, Wattan J, Venables GS, Griffiths PD. Comparison of Doppler ultrasound, magnetic resonance angiographic techniques and catheter angiography in evaluation of carotid stenosis. Clin Radiol. 2000;55(12):912-20.

17- Jungquist G, Nilsson JA. Increase body weight in men after the age of 55 is a risk factor for internal carotid artery stenosis: an epidemiology study of men aged 69. Clinical Psysiology. 1994;14(1):71-7.

18- Kamikawa RS, Fenyo-Pereira M, Fernandes A, Meurer MT. Study of the localization of radiopacities similar to calcified carotid atheroma by means of panoramic radiography. Oral Surg Oral Med Oral Pathol Oral Radiol Endod. 2006;101(3):374-8.

19- Koga M, Kimura K, Minematsu K, Yamaguchi T. Diagnosis of internal carotid artery stenosis greater than $70 \%$ with power Doppler duplex sonography. AJNR Am J Neuroradiol. 2001;22(2):413-7.

20- Langlais RP, Langland OE, Nortjé CJ.. Diagnosis imaging of jaws. Malvern: Willians \& Willians; 1995.

21- Langland OE, Langlais RP, McDavid WD, Delbalso AM. Panoramic radiology. 2nd ed. Philadelphia: Lea \& Febiger; 1989. 440p.

22- Lewis DA, Brooks SL. Carotid artery calcification in general dental population: a retrospective study of panoramic radiographs. Gen Dent. 1999;47(1):98-103.

23- Madden RP, Hodges JS, Salmen CW, Rindal DB, Tunio J, Michalowicz BS, et al. Utility of panoramic radiographs in detecting cervical calcified carotid atheroma. Oral Surg Oral Med Oral Pathol Oral Radiol Endod 2007;103(4):543-8.

24- Moneta GL, Edwards JM, Chitwood RW, Taylor Jr LM, Lee RW, Cummings CA, et al. Correlation of North American Symptomatic Carotid Endarterectomy Trial (NASCET) angiographic definition of $70 \%$ to $90 \%$ internal carotid stenosis with duplex scanning. J Vasc Surg. 1993;17(1):152-

25- Rossouw JE. Lipid-lowering interventions in angiographic trials. Am J Cardiol. 1995;76(9):86-92. 